Supporting Information 


\section{Surface Atomic Decoration of a Manganite to a Modulable Oxygen Evolution Reaction}

Ji Qi ${ }^{\mathrm{a}, \mathrm{b}, 1}$, Huan Liu ${ }^{\mathrm{a}, \mathrm{b}, 1}$, Hang $\mathrm{Xu}^{\mathrm{a}, \mathrm{b}}$, Linglong $\mathrm{Hu}^{\mathrm{b}}$, Chen Wang ${ }^{\mathrm{b}}$, Yuan Zhang ${ }^{\mathrm{b}}$, Ming Feng ${ }^{\mathrm{b}, *}$, and Weiming Lü $\ddot{\mathrm{a}}^{\mathrm{a}, \mathrm{b}, *}$

${ }^{a}$ Condensed Matter Science and Technology Institute, School of Instrumentation Science and Engineering, Harbin Institute of Technology, Harbin 150080, China.

${ }^{b}$ Key Laboratory of Functional Materials Physics and Chemistry of the Ministry of Education, Jilin Normal University, Changchun 130103, China.

${ }^{*}$ Corresponding authors.

E-mail addresses: mingfeng@jlnu.edu.cn (M. Feng) and weiminglv@hit.edu.cn (W. M.

${ }^{1}$ These authors contributed equally to this work.

As we know that the Curie temperature is sensitive to stoichiometry owing to the ferromagnetism/conductivity of LSMO comes from the double-exchange coupling of two neighboring $\mathrm{Mn}$ sites mediating by $\mathrm{O} 2 p$ orbit. So we believe that the oxygen vacancy could not be dominant in LSMO OER performance. To test the stability of LSMO in OER measurements, the magnetic states of as-growth and spent LSMO (001) are measured by the Superconductor Quantum Interface Device (SQUID), the obtained results are shown in Fig. S1. Here we can find that either saturation magnetization or Curie temperature of LSMO remain compared to the as-growth LSMO suggesting that LSMO is a stable catalyst in the OER process. 
Fig. S2 exhibits the TEM pattern of the LSMO/STO oriented-(001) heterostructure. The interface and crystallinity are visible of the heterostructure, which manifests that the high quality LSMO can be fabricated epitaxially on the STO by our Laser-MBE. Within the detection range, no obvious defects and dislocations are found.

The LSMO crystal structure was illustrated by the XRD results, and obtained patterns are shown in Fig. S3. All the LSMO thin films deposited on STOs exhibit a nice epitaxial growth and diffractive peaks of impurity are not observed.

The Tafel slopes are usually employed to present the reaction kinetics of OER. The Tafel plots based polarization curves of LSMOs are exhibited in Fig. S4(a,b). The LSMO (111) exhibits a minimum Tafel plot value of $130.79 \mathrm{mV} / \mathrm{dec}$ and the Tafel plot of LSMO with narrow terrace width is $166.25 \mathrm{mV} / \mathrm{dec}$. The conclusion from the Tafel plots is consistent with the one in the main text.

Fig. S5 shows the 48-hour stability test (I-T) under different applied voltages of LSMO (001). Under different applied voltages, currents can be stabilized in long-term duration.

Electrochemical impedance spectroscopy (EIS) is employed to evaluate the surface of LSMO on the charge transfer efficiency in OER processes. The EIS equivalent circuit diagram fitting data is shown in Fig. S6. The series resistance (Rs) mainly derives from the $\mathrm{KOH}$ solution and the charge transfer resistance (Rct) is strongly relevant to the electrode kinetics, small Rct value represents a faster OER kinetics at the solution-electrocatalyst interface. The double layer capacitance during the OER condition is described by the constant phase element (CPE). 
Fig. S7(a) shows the current density as a function of voltage scan rate. Fig. S7(b) exhibits the double-layer capacitance of LSMO versus the Mn and terrace densities. It follows that LSMOs with high Mn density and inflection Mn possess electrochemically effective surface area (ECSA). All the results demonstrate that inflection Mn sites on the LSMO surface is more beneficial for OER performance.

\section{Figures}

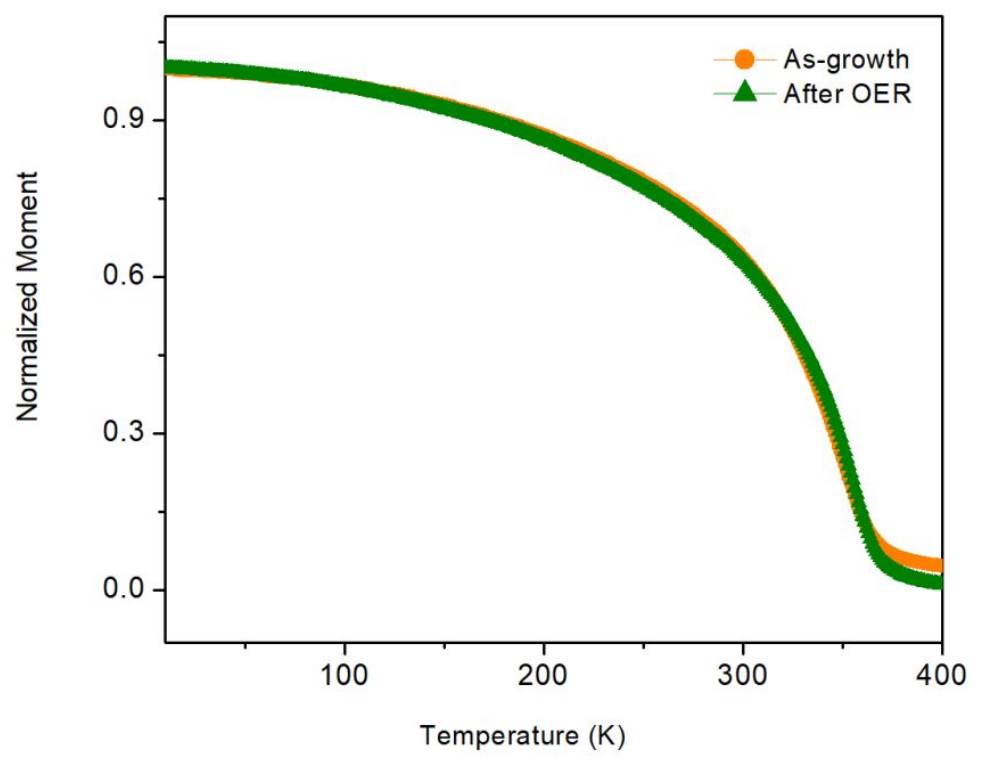

Figure S1. The magnetization versus temperature curves of as-growth LSMO (001) and the spent one after the OER measurement. 


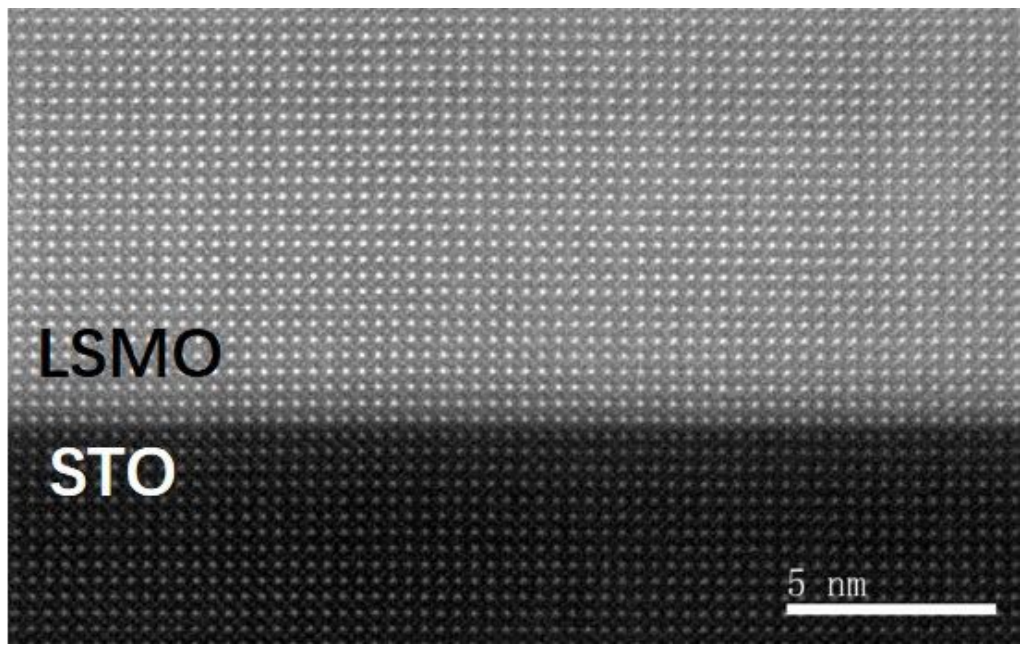

Figure S2. The TEM image of LSMO/STO (001) heterostructure.
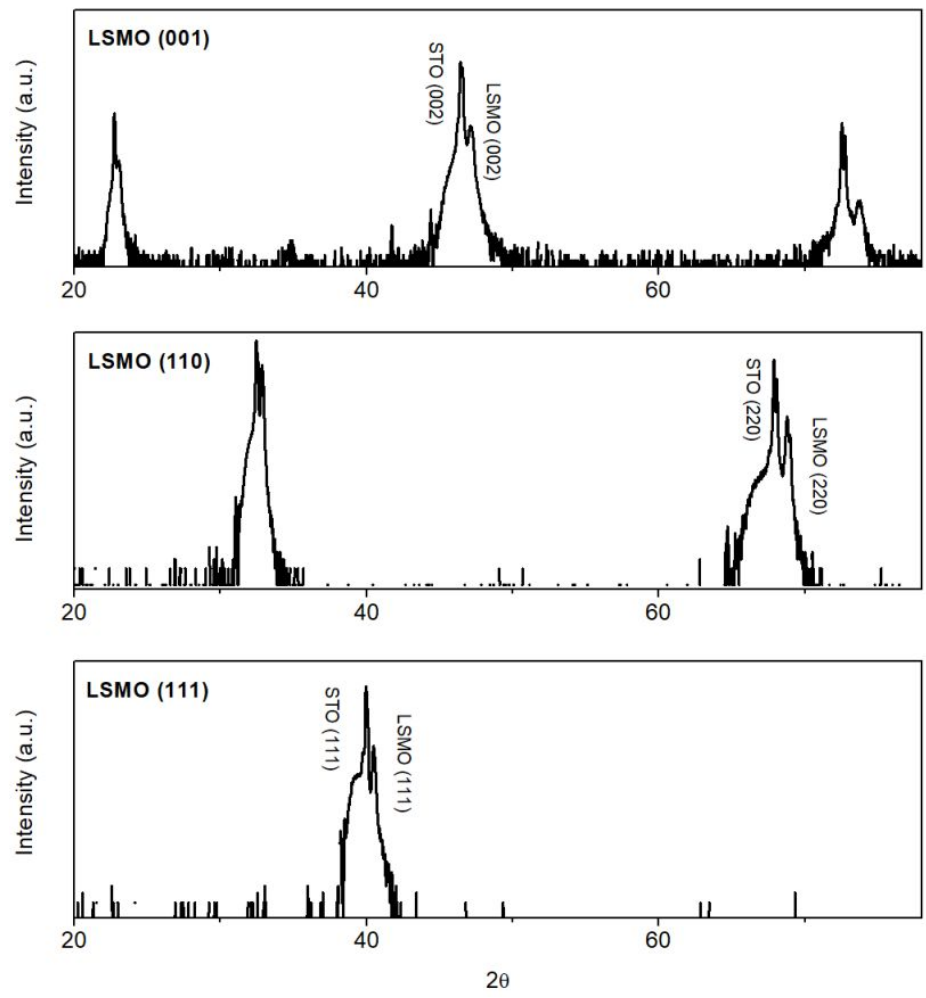

Figure S3. The XRD patterns of LSMO on different STO substrates with various orientations (001), (110), and (111). 
a

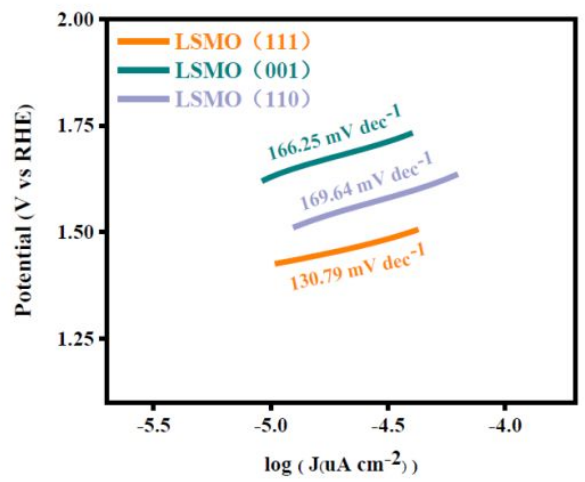

b

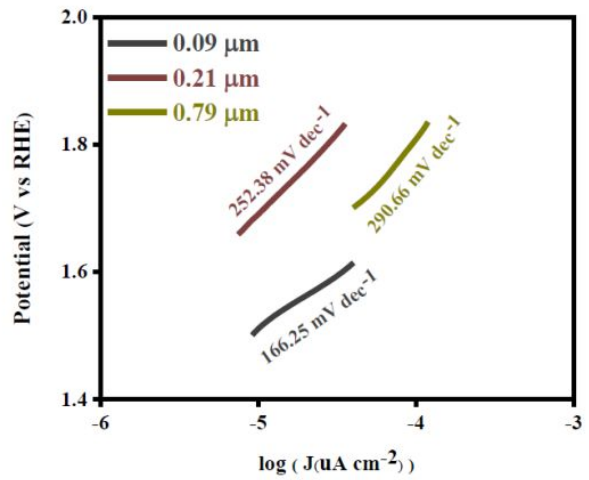

Figure S4. (a) Tafel plots of LSMO with various orientations (001), (110), and (111). (b) Tafel plots of LSMO with different terrace widths.

a

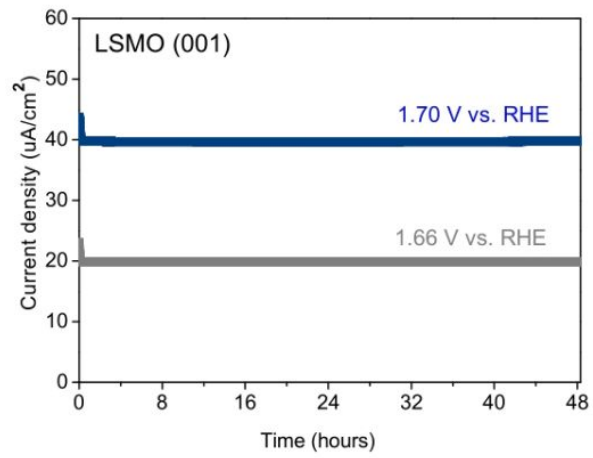

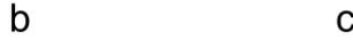

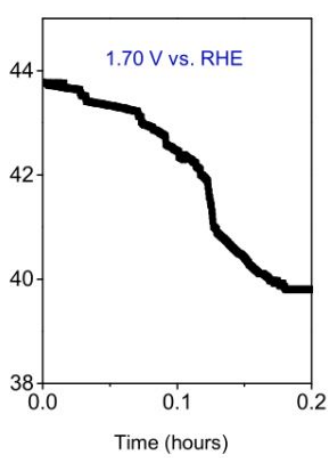

C

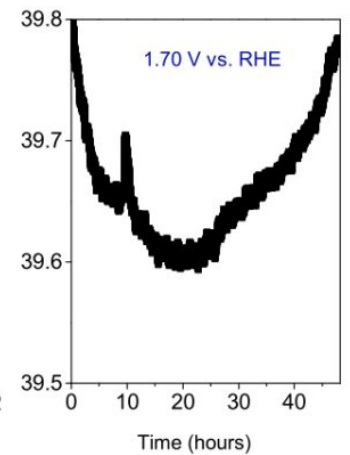

Figure S5. (a), the current density as a function of time at different voltages of 1.70 and $1.66 \mathrm{~V}$ vs.

RHE. The CA curves of $1.7 \mathrm{~V}$ vs. RHE at the beginning regime are shown in (b). (c) shows the enlarged CA curve of $1.7 \mathrm{~V}$ vs. RHE. 


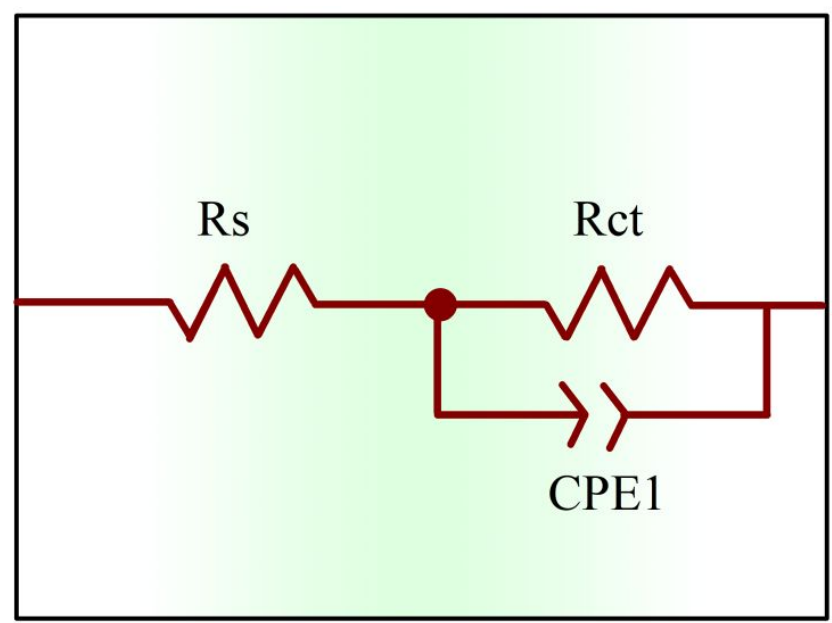

Fig. S6. The EIS equivalent circuit diagram fitting.
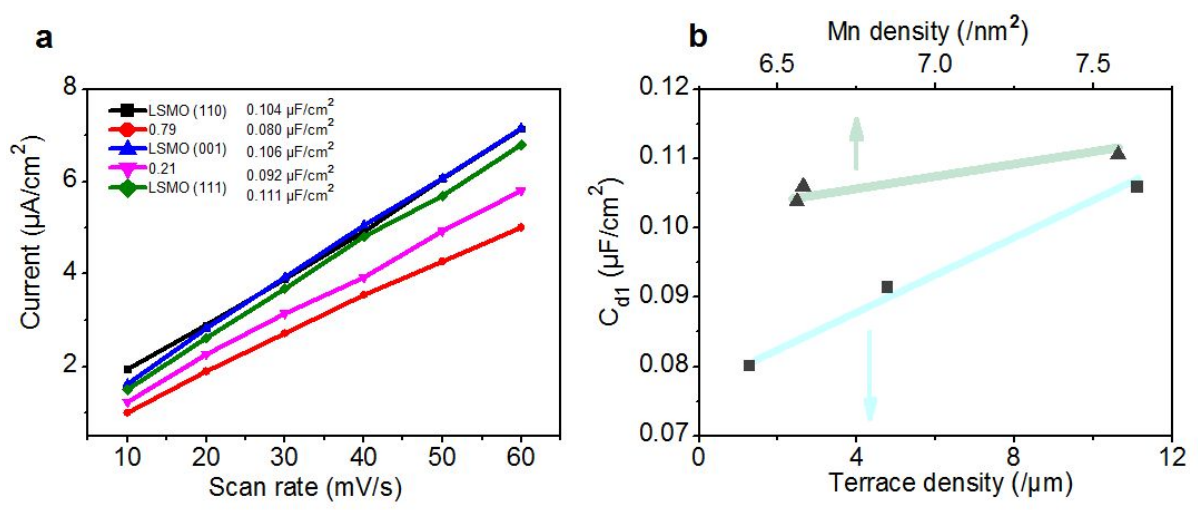

Figure S7. (a), the current density as a function of voltage scan rate. (b), the double-layer capacitance of LSMO versus the Mn and terrace densities. 